\title{
Precision tests of CPT symmetry and Quantum coherence with entangled neutral $\mathrm{K}$
}

\section{Antonio Di Domenico*}

Sapienza University of Rome, and INFN sez. Roma, Italy

E-mail: antonio.didomenicoeromal.infn.it

The neutral kaon system at a $\phi$-factory offers a unique possibility to perform fundamental tests of $C P T$ invariance, as well as of the basic principles of quantum mechanics. The most recent and significant limits on $C P T$ violation are reviewed, including the ones related to possible decoherence mechanisms or Lorentz symmetry breaking, which might be induced in a quantum gravity scenario. The present experimental results show no deviations from the expectations of quantum mechanics and $C P T$ symmetry, while the accuracy in some cases reaches the interesting Planck scale region. The KLOE-2 experiment, presently taking data at the DAФNE facility, will further improve these results.

Corfu Summer Institute 2016 "School and Workshops on Elementary Particle Physics and Gravity" 31 August - 23 September, 2016

Corfu, Greece

\footnotetext{
* Speaker.
} 


\section{Introduction}

The three discrete symmetries of quantum mechanics, $C$ (charge conjugation), $P$ (parity), and $T$ (time reversal) are known to be violated in nature, both singly and in any bilinear combination. Only the combination of the three - CPT (in any order) - appears to be an exact symmetry of nature.

A rigorous proof of the $C P T$ theorem can be found in Refs [1,2,3,4]; it ensures that exact $C P T$ invariance holds for any quantum field theory assuming (1) Lorentz invariance, (2) Locality, and (3) Unitarity (i.e. conservation of probability). Testing the validity of $C P T$ invariance therefore probes the most fundamental assumptions of our present understanding of particles and their interactions, thus strongly motivating both theoretical and experimental investigations on this subject.

The neutral kaon doublet is one of the most intriguing systems in nature. During its time evolution a neutral kaon oscillates between its particle and antiparticle states with a beat frequency $\Delta m \approx 5.3 \times 10^{9} \mathrm{~s}^{-1}$, where $\Delta m$ is the small mass difference between the exponentially decaying states $\mathrm{K}_{\mathrm{L}}$ and $\mathrm{K}_{\mathrm{S}}$. The fortunate coincidence that $\Delta m$ is about half the decay width of $\mathrm{K}_{\mathrm{S}}$ makes it possible to observe a variety of intricate interference phenomena in the production and decay of neutral kaons. In turn, such observations enable us to test quantum mechanics, the interplay of different conservation laws and the validity of various symmetry principles. In particular the extreme sensitivity of the neutral kaon system to a variety of $C P T$-violating effects makes it one of the best candidates for an accurate experimental test of this symmetry [5]. As a figure of merit, the fractional mass difference $\left(m_{\mathrm{K}^{0}}-m_{\overline{\mathrm{K}}^{0}}\right) / m_{\mathrm{K}^{0}}$ can be considered: it can be measured at the level of $\mathscr{O}\left(10^{-18}\right)$ for neutral kaons, while, for comparison, a limit of $\mathscr{O}\left(10^{-14}\right)$ can be reached on the corresponding quantity for the $\mathrm{B}^{0}-\overline{\mathrm{B}}^{0}$ system, and only of $\mathscr{O}\left(10^{-8}\right)$ for proton-antiproton [6].

\section{CPT test from unitarity}

The real part of the complex parameter $\delta$, describing $C P T$ violation in $\mathrm{K}^{0}-\overline{\mathrm{K}}^{0}$ mixing, has been measured by the CPLEAR collaboration studying the time behaviour of semileptonic decays from initially tagged $\mathrm{K}^{0}$ and $\overline{\mathrm{K}}^{0}$ mesons [7]:

$$
\Re \delta=\left(0.30 \pm 0.33_{\text {stat }} \pm 0.06_{\text {syst }}\right) \times 10^{-3} .
$$

One of the most precise and significant tests of the CPT symmetry comes from the unitarity relation, originally derived by Bell and Steinberger [8]:

$$
\begin{aligned}
& \left(\frac{\Gamma_{S}+\Gamma_{L}}{\Gamma_{S}-\Gamma_{L}}+i \tan \phi_{S W}\right)\left[\frac{\Re \varepsilon}{1+|\varepsilon|^{2}}-i \mathfrak{I} \delta\right] \\
& =\frac{1}{\Gamma_{S}-\Gamma_{L}} \sum_{f} A^{*}\left(K_{S} \rightarrow f\right) A\left(K_{L} \rightarrow f\right) \\
& \equiv \sum_{f} \alpha_{f}
\end{aligned}
$$

where $\varepsilon$ is the usual complex parameter describing $C P$ violation in $\mathrm{K}^{0}-\overline{\mathrm{K}}^{0}$ mixing; $\Gamma_{S}$ and $\Gamma_{L}$ are the widths of the physical states $\mathrm{K}_{\mathrm{S}}$ and $\mathrm{K}_{\mathrm{L}} ; \phi_{S W}$ is the superweak phase; $A\left(K_{i} \rightarrow f\right)$ is the decay amplitude of the state $K_{i}$ into final state $f$, and the sum runs over all possible final states. 
The above relationship can be used to bound the parameter $\mathfrak{I} \delta$, after having provided all the $\alpha_{i}$ parameters, $\Gamma_{S}, \Gamma_{L}$, and $\phi_{S W}$ as inputs. Using several measurements from different experiments (KLOE, CPLEAR, NA48, KTEV etc.) the following result is obtained [6, 9]:

$$
\mathfrak{I} \delta=(-0.7 \pm 1.4) \times 10^{-5},
$$

which is the most stringent limit on $\mathfrak{I} \delta$. The main limiting factors of this result are the uncertainties on $\pi \pi$ and semileptonic decay amplitudes.

The limits on $\mathfrak{I} \delta$ and $\mathfrak{R} \delta$ can be used to constrain the mass and width difference between $\mathrm{K}^{0}$ and $\overline{\mathrm{K}}^{0}$. In the limit $\Gamma_{\mathrm{K}^{0}}-\Gamma_{\overline{\mathrm{K}}^{0}}=0$, i.e. neglecting $C P T$-violating effects in the decay amplitudes, the best bound on the neutral kaon mass difference is obtained [6]:

$$
\left|m_{\mathrm{K}^{0}}-m_{\overline{\mathrm{K}}^{0}}\right|<4.0 \times 10^{-19} \mathrm{GeV} \quad \text { at } 95 \% \mathrm{CL} .
$$

\section{Quantum coherence and CPT tests}

DA $\Phi N E$, the Frascati $\phi$-factory [10], is an $e^{+} e^{-}$collider working at a center of mass energy of $\sqrt{s} \sim 1020 \mathrm{MeV}$, corresponding to the peak of the $\phi$ resonance. The $\phi$ production cross section is $\sim 3 \mu \mathrm{b}$, and its decay into $\mathrm{K}^{0} \overline{\mathrm{K}}^{0}$ has a branching fraction of $34 \%$. The neutral kaon pair is produced in a coherent quantum state with quantum numbers $J^{P C}=1^{--}$:

$$
|i\rangle=\frac{1}{\sqrt{2}}\left\{\left|\mathrm{~K}^{0}\right\rangle\left|\overline{\mathrm{K}}^{0}\right\rangle-\left|\overline{\mathrm{K}}^{0}\right\rangle\left|\mathrm{K}^{0}\right\rangle\right\}=\frac{\mathscr{N}}{\sqrt{2}}\left\{\left|\mathrm{~K}_{\mathrm{S}}\right\rangle\left|\mathrm{K}_{\mathrm{L}}\right\rangle-\left|\mathrm{K}_{\mathrm{L}}\right\rangle\left|\mathrm{K}_{\mathrm{S}}\right\rangle\right\}
$$

where $\mathscr{N}=\sqrt{\left(1+\left|\varepsilon_{S}\right|^{2}\right)\left(1+\left|\varepsilon_{L}\right|^{2}\right)} /\left(1-\varepsilon_{S} \varepsilon_{L}\right) \simeq 1$ is a normalization factor, and $\varepsilon_{S, L}=\varepsilon \pm \delta$.

The observable quantity is the double differential decay rate of the state $|i\rangle$ into decay products $f_{1}$ and $f_{2}$ at proper times $t_{1}$ and $t_{2}$, respectively. After integration on $\left(t_{1}+t_{2}\right)$ at fixed time difference $\Delta t=t_{1}-t_{2}$, the decay intensity can be written as follows [5]:

$$
\begin{gathered}
I\left(f_{1}, f_{2} ; \Delta t \geq 0\right)=C_{12}\left\{\left|\eta_{1}\right|^{2} e^{-\Gamma_{L} \Delta t}+\left|\eta_{2}\right|^{2} e^{-\Gamma_{S} \Delta t}\right. \\
\left.-2\left|\eta_{1}\right|\left|\eta_{2}\right| e^{-\frac{\left(\Gamma_{S}+\Gamma_{L}\right)}{2} \Delta t} \cos \left[\Delta m \Delta t+\phi_{2}-\phi_{1}\right]\right\} .
\end{gathered}
$$

This expression is valid for $\Delta t \geq 0$, while for $\Delta t<0$ the substitutions $\Delta t \rightarrow|\Delta t|$ and $1 \leftrightarrow 2$ have to be applied, and with $\Delta m=m_{L}-m_{S}$,

$$
\begin{aligned}
\eta_{i} & \equiv\left|\eta_{i}\right| e^{i \phi_{i}}=\frac{\left\langle f_{i}|T| \mathrm{K}_{\mathrm{L}}\right\rangle}{\left\langle f_{i}|T| \mathrm{K}_{\mathrm{S}}\right\rangle}, \\
C_{12} & =\frac{|\mathscr{N}|^{2}}{2\left(\Gamma_{S}+\Gamma_{L}\right)}\left|\left\langle f_{1}|T| \mathrm{K}_{\mathrm{S}}\right\rangle\left\langle f_{2}|T| \mathrm{K}_{\mathrm{S}}\right\rangle\right|^{2} .
\end{aligned}
$$

Due to the huge difference in the lifetimes of the physical states $\left(\tau_{L} \gg \tau_{S}\right.$ ), for $t_{1} \gg t_{2}, \tau_{S}$ (or $t_{2} \gg t_{1}, \tau_{S}$ ) the decay intensity in eq.(3.2) behaves like the initial state were an incoherent mixture of states $\left|\mathrm{K}_{\mathrm{S}}\right\rangle\left|\mathrm{K}_{\mathrm{L}}\right\rangle$ and $\left|\mathrm{K}_{\mathrm{L}}\right\rangle\left|\mathrm{K}_{\mathrm{S}}\right\rangle$. Hence the detection of a kaon at large times tags a $\mathrm{K}_{\mathrm{S}}$ in the opposite direction. This is a unique feature at a $\phi$-factory, not possible at fixed target experiments, that can be exploited to select very pure $\mathrm{K}_{\mathrm{S}}$ beams. 
The KLOE experiment at DAФNE completed its first data taking campaign in March 2006 with a total integrated luminosity $\mathrm{L} \sim 2.5 \mathrm{fb}^{-1}$, corresponding to $\sim 7.5 \times 10^{9} \phi$-mesons produced.

The KLOE detector consists mainly of a large volume drift chamber [11] surrounded by an electromagnetic calorimeter [12]. A superconducting coil provides a $0.52 \mathrm{~T}$ solenoidal magnetic field. At KLOE a $\mathrm{K}_{\mathrm{S}}$ is tagged by identifying the interaction of the $\mathrm{K}_{\mathrm{L}}$ in the calorimeter $\left(\mathrm{K}_{\mathrm{L}^{-}}\right.$ crash), while a $\mathrm{K}_{\mathrm{L}}$ is tagged by detecting a $\mathrm{K}_{\mathrm{S}} \rightarrow \pi^{+} \pi^{-}$decay near the interaction point (IP).

The quantum interference between the two kaons initially in the entangled state in eq.(3.1) and decaying in the $C P$ violating channel $\phi \rightarrow \mathrm{K}_{\mathrm{S}} \mathrm{K}_{\mathrm{L}} \rightarrow \pi^{+} \pi^{-} \pi^{+} \pi^{-}$, has been observed for the first time by the KLOE collaboration [13]. The measured $|\Delta t|$ distribution can be fitted with the distribution:

$$
I\left(\pi^{+} \pi^{-}, \pi^{+} \pi^{-} ;|\Delta t|\right) \propto e^{-\Gamma_{L}|\Delta t|}+e^{-\Gamma_{S}|\Delta t|}-2\left(1-\zeta_{S L}\right) e^{-\frac{\left(\Gamma_{S}+\Gamma_{L}\right)}{2}|\Delta t|} \cos (\Delta m|\Delta t|),
$$

where the quantum mechanical expression in the $\left\{\mathrm{K}_{\mathrm{S}}, \mathrm{K}_{\mathrm{L}}\right\}$ basis has been modified with the introduction of a decoherence parameter $\zeta_{S L}$, and a factor $\left(1-\zeta_{S L}\right)$ multiplying the interference term. Analogously, a $\zeta_{0 \overline{0}}$ parameter can be defined in the $\left\{\mathrm{K}^{0}, \overline{\mathrm{K}}^{0}\right\}$ basis [14]. After having included resolution and detection efficiency effects, having taken into account the background due to coherent and incoherent $\mathrm{K}_{\mathrm{S}}$-regeneration on the beam pipe wall, the small contamination of non-resonant $e^{+} e^{-} \rightarrow \pi^{+} \pi^{-} \pi^{+} \pi^{-}$events, and keeping $\Delta m, \Gamma_{S}$ and $\Gamma_{L}$ fixed to the PDG values, the fit is performed on the $|\Delta t|$ distribution. The analysis of a data sample corresponding to $\mathrm{L} \sim 1.5 \mathrm{fb}^{-1}$ yields the following results $[13,15,16]$ :

$$
\begin{aligned}
& \zeta_{S L}=\left(0.3 \pm 1.8_{\text {stat }} \pm 0.6_{\text {syst }}\right) \times 10^{-2} \\
& \zeta_{0 \overline{0}}=\left(1.4 \pm 9.5_{\text {stat }} \pm 3.8_{\text {syst }}\right) \times 10^{-7}
\end{aligned}
$$

compatible with the prediction of quantum mechanics, i.e. $\zeta_{S L}=\zeta_{0 \overline{0}}=0$, and no decoherence effect. In particular the result on $\zeta_{0 \overline{0}}$ has a high precision, $\mathscr{O}\left(10^{-6}\right)$, due to the $C P$ suppression present in the specific decay channel; it is an improvement by five orders of magnitude over the previous limit, obtained by Bertlmann and co-workers [14] in a re-analysis of CPLEAR data [17]. This result can also be compared to a similar one recently obtained in the B meson system [18], where an accuracy of $\mathscr{O}\left(10^{-2}\right)$ has been reached.

At a microscopic level, in a quantum gravity picture, space-time might be subjected to inherent non-trivial quantum metric and topology fluctuations at the Planck scale $\left(\sim 10^{-33} \mathrm{~cm}\right)$, called generically space-time foam, with associated microscopic event horizons. This space-time structure would lead to pure states evolving to mixed states, i.e. the decoherence of apparently isolated matter systems [19]. This decoherence, in turn, necessarily implies, by means of a theorem [20], $C P T$ violation, in the sense that the quantum mechanical operator generating $C P T$ transformations is ill-defined.

A model for decoherence can be formulated [21, 22] in which neutral kaons are described by a density matrix $\rho$ that obeys a modified Liouville-von Neumann equation. In this context $\gamma$ is one of the relevant parameters signalling decoherence and $C P T$ violation. It has mass units and in a Quantum Gravity scenario it is presumed to be at most of $\mathscr{O}\left(m_{K}^{2} / M_{\text {Planck }}\right) \sim 2 \times 10^{-20} \mathrm{GeV}$. The KLOE collaboration, studying the same $I\left(\pi^{+} \pi^{-}, \pi^{+} \pi^{-} ;|\Delta t|\right)$ distribution as in the $\zeta$ parameters 
analysis, obtained the following result $[13,15,16]$ :

$$
\gamma=\left(0.7 \pm 1.2_{\text {stat }} \pm 0.3_{\text {syst }}\right) \times 10^{-21} \mathrm{GeV},
$$

compatible with no CPT violation, improving the previous result by CPLEAR [23], while the sensitivity reaches the interesting region.

As discussed above, in a quantum gravity framework inducing decoherence, the $C P T$ operator is ill-defined. This consideration has intriguing consequences in correlated neutral kaon states, where the resulting loss of particle-antiparticle identity could induce a breakdown of the correlation in state (3.1) imposed by Bose statistics [24, 25]. As a result the initial state (3.1) can be parametrized in general as:

$$
|i\rangle=\frac{1}{\sqrt{2}}\left[\left|\mathrm{~K}^{0}\right\rangle\left|\overline{\mathrm{K}}^{0}\right\rangle-\left|\overline{\mathrm{K}}^{0}\right\rangle\left|\mathrm{K}^{0}\right\rangle+\omega\left(\left|\mathrm{K}^{0}\right\rangle\left|\overline{\mathrm{K}}^{0}\right\rangle+\left|\overline{\mathrm{K}}^{0}\right\rangle\left|\mathrm{K}^{0}\right\rangle\right)\right]
$$

where $\omega$ is a complex parameter describing a novel $C P T$ violation phenomenon, and in this scenario its order of magnitude is expected to be at most:

$$
|\omega| \sim\left[\left(m_{K}^{2} / M_{\text {Planck }}\right) / \Delta \Gamma\right]^{1 / 2} \sim 10^{-3}
$$

with $\Delta \Gamma=\Gamma_{S}-\Gamma_{L}$. An analysis performed by the KLOE collaboration on the $C P$ violating $\phi \rightarrow$ $\mathrm{K}_{\mathrm{S}} \mathrm{K}_{\mathrm{L}} \rightarrow \pi^{+} \pi^{-} \pi^{+} \pi^{-}$events, including in the fit of the $\Delta t$ distribution the modified initial state eq.(3.8), yields the most precise measurement of the complex parameter $\omega[13,15,16]$ :

$$
\begin{aligned}
& \mathfrak{R}(\omega)=\left(-1.6_{-2.1}^{+3.0} \text { stat }^{+} \pm 0.4_{\text {syst }}\right) \times 10^{-4} \\
& \mathfrak{I}(\omega)=\left(-1.7_{-3.0_{\text {stat }}}^{+3.3} \pm 1.2_{\text {syst }}\right) \times 10^{-4},
\end{aligned}
$$

with an accuracy that already reaches the interesting Planck scale region.

\section{CPT- and Lorentz-symmetry tests}

$C P T$ invariance holds for any realistic Lorentz-invariant quantum field theory. However a very general theoretical possibility for $C P T$ violation is based on spontaneous breaking of Lorentz symmetry $[26,27,28]$, which appears to be compatible with the basic tenets of quantum field theory and retains the property of gauge invariance and renormalizability (Standard Model Extensions $\mathrm{SME}$ ). In the SME for neutral kaons, $C P T$ violation manifests to lowest order only in the mixing parameter $\delta$, (e.g., vanishes at first order in the decay amplitudes), and exhibits a dependence on the 4-momentum of the kaon:

$$
\delta \approx i \sin \phi_{S W} e^{i \phi_{S W}} \gamma_{K}\left(\Delta a_{0}-\overrightarrow{\beta_{K}} \cdot \Delta \vec{a}\right) / \Delta m
$$

where $\gamma_{K}$ and $\overrightarrow{\beta_{K}}$ are the kaon boost factor and velocity in the observer frame, $\phi_{S W}$ is the so called superweak phase, and $\Delta a_{\mu}$ are four $C P T$ - and Lorentz-violating coefficients for the two valence quarks in the kaon. 
By studying the $\Delta t$ interference pattern of the entangled neutral kaon pairs in the decay channel $\phi \rightarrow \mathrm{K}_{\mathrm{S}} \mathrm{K}_{\mathrm{L}} \rightarrow \pi^{+} \pi^{-} \pi^{+} \pi^{-}$, as a function of sidereal time and particle direction in celestial coordinates, the KLOE collaboration obtained the following results [29]:

$$
\begin{aligned}
& \Delta a_{0}=\left(-6.0 \pm 7.7_{\text {stat }} \pm 3.1_{\text {syst }}\right) \times 10^{-18} \mathrm{GeV} \\
& \Delta a_{X}=\left(0.9 \pm 1.5_{\text {stat }} \pm 0.6_{\text {syst }}\right) \times 10^{-18} \mathrm{GeV} \\
& \Delta a_{Y}=\left(-2.0 \pm 1.5_{\text {stat }} \pm 0.5_{\text {syst }}\right) \times 10^{-18} \mathrm{GeV} \\
& \Delta a_{Z}=\left(3.1 \pm 1.7_{\text {stat }} \pm 0.5_{\text {syst }}\right) \times 10^{-18} \mathrm{GeV}
\end{aligned}
$$

These results constitute the most sensitive measurements in the quark sector of the SME, and can be compared to similar results obtained in the $B$ and $D$ meson systems, where an accuracy of $\mathscr{O}\left(10^{-15} \mathrm{GeV}\right)$ and $\mathscr{O}\left(10^{-13} \mathrm{GeV}\right)$, respectively, has been reached [30, 31].

\section{Direct CPT tests in transitions}

In Sect. 2 the best limits on the $\delta$ parameter expressing $C P T$ violation in the $\mathrm{K}^{0}-\overline{\mathrm{K}}^{0}$ mixing matrix, i.e. in the standard Weisskopf-Wigner approach [32], have been reviewed, yielding a stringent limit on the difference of masses $\left(m_{\mathrm{K}^{0}}-m_{\overline{\mathrm{K}}^{0}}\right)$. However the $C P T$ violating probe is often limited to such a difference (of masses or other intrinsic properties) for a particle and its anti-particle, i.e. to diagonal mass terms. In many physical phenomena the perturbing effect does not appear at first order in perturbation theory: it would be sufficient that the perturbation breaks a symmetry of the non-perturbed states. This vanishing effect at first order for the diagonal elements, like e.g. the case of the electric dipole moment for $T$ violation, is not present for transitions (non-diagonal elements) [33].

Recently a novel kind of $C P T$ test for transitions in the neutral kaon system has been proposed [34], where the exchange of in and out states (and CP conjugation), required for a direct and genuine $C P T$ test, is performed exploiting the entanglement of the kaon pair produced at a $\phi$-factory. This methodology has been proposed for a direct test of the $T$ symmetry in the same context [35], similarly to the one adopted for the performed test in the B meson system at Bfactories [33, 36, 37]. The decay is not an essential ingredient for a non-vanishing effect and it is only used for filtering the appropriate initial and final states of the neutral kaon transition [38]. Explicitly, in the standard Weisskopf-Wigner approach to this system, the $C P T$-violating effects can be connected to the $\Re \delta$ parameter, a genuine $C P T$-violating effect independent of $\Delta \Gamma$.

In order to implement this test, the Einstein-Podolsky-Rosen (EPR) entanglement of neutral mesons produced at a $\phi$-factory must be exploited. In fact the perfect anticorrelation of the state in Eq. (3.1), implies that it is possible to have a "flavor-tag"or a "CP-tag", i.e. to infer the flavor $\left(\mathrm{K}^{0}\right.$ or $\left.\overline{\mathrm{K}}^{0}\right)$ or the $C P\left(\mathrm{~K}_{+}\right.$or $\left.\mathrm{K}_{-}\right)$state of the still alive kaon by observing a specific flavor decay $\left(\pi^{+} \ell^{-} v\right.$ or $\pi^{-} \ell^{+} \bar{v}$ ) or CP decay ( $\pi \pi$ or $\left.\pi^{0} \pi^{0} \pi^{0}\right)$ of the other (and first decaying) kaon in the pair.

In this way one can experimentally access - for instance - the transition $\mathrm{K}^{0} \rightarrow \mathrm{K}_{+}$, taken as reference, and the $\mathrm{K}_{+} \rightarrow \mathrm{K}^{0}, \overline{\mathrm{K}}^{0} \rightarrow \mathrm{K}_{+}$and $\mathrm{K}_{+} \rightarrow \overline{\mathrm{K}}^{0}$ transitions, i.e. the $T, C P$ and $C P T$ conjugated transitions, respectively. Specifically for the CPT symmetry test, one can directly compare the probabilities for the reference transition and the $C P T$ conjugated one through the following ratios 
of probabilities:

$$
\begin{aligned}
& R_{1, C P T}(\Delta t)=P\left[\mathrm{~K}_{+}(0) \rightarrow \overline{\mathrm{K}}^{0}(\Delta t)\right] / P\left[\mathrm{~K}^{0}(0) \rightarrow \mathrm{K}_{+}(\Delta t)\right] \\
& R_{2, C P T}(\Delta t)=P\left[\mathrm{~K}^{0}(0) \rightarrow \mathrm{K}_{-}(\Delta t)\right] / P\left[\mathrm{~K}_{-}(0) \rightarrow \overline{\mathrm{K}}^{0}(\Delta t)\right] \\
& R_{3, C P T}(\Delta t)=P\left[\mathrm{~K}_{+}(0) \rightarrow \mathrm{K}^{0}(\Delta t)\right] / P\left[\overline{\mathrm{K}}^{0}(0) \rightarrow \mathrm{K}_{+}(\Delta t)\right] \\
& R_{4, C P T}(\Delta t)=P\left[\overline{\mathrm{K}}^{0}(0) \rightarrow \mathrm{K}_{-}(\Delta t)\right] / P\left[\mathrm{~K}_{-}(0) \rightarrow \mathrm{K}^{0}(\Delta t)\right] .
\end{aligned}
$$

The measurement of any deviation from the prediction $R_{i, C P T}(\Delta t)=1$ imposed by $C P T$ invariance is a signal of $C P T$ violation. It is worth noting that for $\Delta t=0$ :

$$
R_{1, C P T}(0)=R_{2, C P T}(0)=R_{3, C P T}(0)=R_{4, C P T}(0)=1 \text {, }
$$

i.e. the $C P T$-violating effect is built in the time evolution of the system, and it is absent at $\Delta t=0$. For $\Delta t \gg \tau_{S}$, assuming the presence of $C P T$ violation only in the mass matrix $(\delta \neq 0)$ and nothing else, one gets:

$$
\begin{aligned}
& R_{2, C P T}\left(\Delta t \gg \tau_{S}\right) \simeq 1-4 \Re \delta \\
& R_{4, C P T}\left(\Delta t \gg \tau_{S}\right) \simeq 1+4 \Re \delta
\end{aligned}
$$

i.e. the $C P T$-violating effect built in the time evolution reaches a "plateau" regime and dominates in this limit. It is a genuine effect because $\Re \delta$ does not depend on $\Delta \Gamma$ as an essential ingredient $[33,38]$.

At a $\phi$-factory one can define two observable ratios:

$$
\begin{gathered}
R_{2, C P T}^{\exp }(\Delta t) \equiv \frac{I\left(\ell^{-}, 3 \pi^{0} ; \Delta t\right)}{I\left(\pi \pi, \ell^{-} ; \Delta t\right)} \\
R_{4, C P T}^{\exp }(\Delta t) \equiv \frac{I\left(\ell^{+}, 3 \pi^{0} ; \Delta t\right)}{I\left(\pi \pi, \ell^{+} ; \Delta t\right)},
\end{gathered}
$$

They are related to the $R_{i, C P T}(\Delta t)$ ratios defined in eqs (5.1) as follows, for $\Delta t \geq 0$ :

$$
\begin{aligned}
& R_{2, C P T}^{\exp }(\Delta t)=R_{2, C P T}(\Delta t) \times D_{C P T} \\
& R_{4, C P T}^{\exp }(\Delta t)=R_{4, C P T}(\Delta t) \times D_{C P T}
\end{aligned}
$$

whereas for $\Delta t<0$ one has:

$$
\begin{aligned}
& R_{2, C P T}^{\exp }(\Delta t)=R_{1, C P T}(|\Delta t|) \times D_{C P T} \\
& R_{4, C P T}^{\exp }(\Delta t)=R_{3, C P T}(|\Delta t|) \times D_{C P T},
\end{aligned}
$$

with

$$
D_{C P T}=\frac{\left|\left\langle 3 \pi^{0}|T| \mathrm{K}_{-}\right\rangle\right|^{2}}{\left|\left\langle\pi \pi|T| \mathrm{K}_{+}\right\rangle\right|^{2}}=\frac{\mathrm{BR}\left(\mathrm{K}_{\mathrm{L}} \rightarrow 3 \pi^{0}\right)}{\mathrm{BR}\left(\mathrm{K}_{\mathrm{S}} \rightarrow \pi \pi\right)} \frac{\Gamma_{L}}{\Gamma_{S}},
$$


where the last r.h.s. equality holds with a high degree of accuracy, at least $\mathscr{O}\left(10^{-7}\right)$. The value of $D_{C P T}$ can be therefore directly evaluated from branching ratios and lifetimes.

The presence of direct $C P$ violation contributions in the decay amplitudes, even though in principle could mimic $C P T$ violation effects, turns out to be totally irrelevant for the plateau region $\Delta t \gg \tau_{S}$ (see detailed description in Ref. [34]). The effect of a possible violation of the $\Delta S=\Delta Q$ rule is also not affecting the $C P T$ test in the same region with the double ratio defined as:

$$
\frac{R_{2, C P T}^{\exp }\left(\Delta t \gg \tau_{S}\right)}{R_{4, C P T}^{\exp }\left(\Delta t \gg \tau_{S}\right)}=1-8 \Re \delta-8 \Re x_{-} .
$$

with $x_{-}$describing $C P T$ violation in the $\Delta S \neq \Delta Q$ semileptonic decay amplitudes. Therefore the double ratio (5.10) constitutes one of the most robust observables for the proposed $C P T$ test. It is independent of $D_{C P T}$, and in the limit $\Delta t \gg \tau_{S}$ it exhibits a pure and genuine $C P T$ violating effect, even without the assumptions of the validity of the $\Delta S=\Delta Q$ rule and of negligible contaminations from direct $C P$ violation.

KLOE and KLOE-2 data are being analysed to get a precise measurement of the two observable ratios $R_{2, C P T}^{\exp }(\Delta t)$ and $R_{4, C P T}^{\exp }(\Delta t)$. A statistical sensitivity on the double ratio (5.10) of $\mathscr{O}\left(10^{-3}\right)$ can be obtained for $L=5 \mathrm{fb}^{-1}$ [34].

\section{The KLOE-2 experiment at $\mathrm{DA} \Phi \mathrm{NE}$}

KLOE-2 represents the continuation of the KLOE experiment with a new physics program mainly focused on the study of $\mathrm{K}_{\mathrm{S}}$ and $\eta$ decays as well as on kaon interferometry and tests of discrete symmetries $[5,39]$. The new data taking campaign aims to collect more than $5 \mathrm{fb}^{-1}$ of integrated luminosity at the upgraded DAФNE, which is implementing an innovative collision scheme based on a crab-waist configuration [40, 41]. The major upgrade of the KLOE detector consists in the addition of an inner tracker [42] for the improvement of the resolution of decay vertices close to the interaction point, therefore improving the sensitivity on several parameters based on kaon interferometry measurements. The KLOE-2 data taking campaign started in November 2014, an integrated luminosity of $3.5 \mathrm{fb}^{-1}$ has been collected by Spring 2017, and the goal of at least $5 \mathrm{fb}^{-1}$ is expected to be reached by Spring 2018 .

\section{Conclusions}

The neutral kaon system constitutes an excellent laboratory for the study of discrete symmetries, and a $\phi$-factory represents a unique opportunity to push forward these studies, being also an ideal place to investigate the entanglement and correlation properties of the produced $\mathrm{K}^{0} \overline{\mathrm{K}}^{0}$ pairs.

The parameters related to several possible CPT violations effects, including decoherence and Lorentz-symmetry breaking effects which might be justified in a quantum-gravity framework, have been measured, in some cases with a precision that very interestingly reaches the Planck scale region.

The KLOE physics program is continuing with the KLOE-2 experiment, presently taking data at the DAФNE facility with an upgraded detector, and significant improvements are expected in all these tests. 


\section{Acknowledgments}

The author would like to warmly thank George Zoupanos and all the organizing committee for the invitation to the Corfu Summer Institute 2016 "School and Workshops on Elementary Particle Physics and Gravity", and the very pleasant stay in Corfu.

\section{References}

[1] G. Lueders, Ann. Phys. (NY) 2 (1957) 1, reprinted in Ann. Phys. (NY) 281 (2000) 1004.

[2] W. Pauli, Exclusion principle, Lorentz group and reflexion of space-time and charge in Niels Bohr and the development of physics, edited by W. Pauli, Pergamon, London, 1955, p.30.

[3] J. S. Bell, Proc. R. Soc. London A 231 (1955) 479

[4] R. Jost, Helv. Phys. Acta 30 (1957) 409.

[5] A. Di Domenico (Editor), Handbook on Neutral Kaon Interferometry at a $\phi$-factory, Frascati Phys. Ser. 43 (2007)

[6] C. Patrignani et al. (Particle Data Group), Chin. Phys. C, 40, 100001 (2016).

[7] A. Angelopoulos et al., CPLEAR collaboration, Phys. Lett. B 444, 52 (1998)

[8] J. S. Bell and J. Steinberger, Proc. of the Oxford Int. Conf. on Elementary Particles (1965)

[9] F. Ambrosino et al., KLOE collaboration, JHEP 12011 (2006).

[10] A. Gallo et al. DAФNE Status Report, CONF.PROC.C060626 (2006) 604-606

[11] M. Adinolfi et al., KLOE collaboration, Nucl. Instr. and Meth. A 488 (2002) 51.

[12] M. Adinolfi et al., KLOE collaboration, Nucl. Instr. and Meth. A 482 (2002) 364.

[13] F. Ambrosino et al., KLOE collaboration, Phys. Lett. B 642 (2006) 315.

[14] R. A. Bertlmann, W. Grimus, B. C. Hiesmayr, Phys. Rev. D 60 (1999) 114032.

[15] A. Di Domenico and the KLOE collaboration, J. Phys. Conf. Ser. 171, 012008 (2009)

[16] A. Di Domenico and the KLOE collaboration, Found. Phys. 40 , 852 (2010)

[17] A. Apostolakis et al., CPLEAR collaboration, Phys. Lett. B 422 (1998) 339.

[18] A. Go et al, Belle collaboration, Phys. Rev. Lett. 99 (2007) 131802.

[19] S. Hawking, Commun. Math. Phys. 87 (1982) 395.

[20] R. Wald, Phys. Rev. D 21 (1980) 2742.

[21] J. Ellis, J. S. Hagelin, D. V. Nanopoulos, M. Srednicki , Nucl. Phys. B 241 (1984) 381.

[22] J. Ellis, J. L. Lopez, N. .E. Mavromatos, D. V. Nanopoulos, Phys. Rev. D 53 (1996) 3846.

[23] R. Adler et al., CPLEAR collaboration, Phys. Lett. B 364 (1995) 239.

[24] J. Bernabeu, N. Mavromatos, J. Papavassiliou, Phys. Rev. Lett. 92 (2004) 131601.

[25] J. Bernabeu, N. Mavromatos, J. Papavassiliou, A. Waldron-Lauda, Nucl. Phys. B 744 (2006) 180.

[26] V. A. Kostelecký, Phys. Rev. Lett. 80, 1818 (1998). 
[27] V. A. Kostelecký, Phys. Rev. D 61, 016002 (1999).

[28] V. A. Kostelecký, Phys. Rev. D 64, 076001 (2001).

[29] D. Babusci et al., Phys. Lett. B 730, 89 (2014).

[30] Data Tables for Lorentz and CPT Violation, V.A. Kostelecký and N. Russell, 2016 edition, arXiv:0801.0287v9.

[31] R. Aaij et al., Phys. Rev. Lett. 116, 241601 (2016).

[32] V. Weisskopf and E. P. Wigner, Z. Phys. 63, 54 (1930)

[33] J. Bernabeu and F. Martinez-Vidal, Rev. Mod. Phys. 87, 165 (2015)

[34] J. Bernabeu, A. Di Domenico, P. Villanueva-Perez, JHEP 10, 139 (2015)

[35] J. Bernabeu, A. Di Domenico, P. Villanueva-Perez, Nucl. Phys. B. 868, 102 (2013)

[36] J. Bernabeu, F. Martínez-Vidal, P. Villanueva-Pérez, JHEP 08, 064 (2012)

[37] J. P. Lees et al., Babar Collaboration, Phys. Rev. Lett. 109, 211801 (2012)

[38] L. Wolfenstein, Int. J. Mod. Phys. E 8, 501 (1999)

[39] G. Amelino-Camelia et al., Eur. Phys. J. C 68, 619 (2010)

[40] M. Zobov et al., Phys. Rev. Lett. 104, 174801 (2010).

[41] C. Milardi et al., JINST, 7, 2012,T03002.

[42] E. De Lucia on behalf of KLOE-2 collaboration, Proceedings of the KLOE-2 Workshop on $e^{+} e^{-}$ collision physics at $1 \mathrm{GeV}$, Frascati, 26-28 October 2016, to appear on Eur. Phys. J. Web of Conferences. 